\title{
Art chorégraphique et sciences, une visite à deux voix des musées de sciences et techniques
}

\author{
Choreographic art and science, a two-voice visit in science and \\ technology museums
}

\author{
Micheline Lelièvre ${ }^{1}$, Isabelle Astic ${ }^{2}$ \\ ${ }^{1}$ chorégraphe, collectif Pignon sur rue \\ ${ }^{2}$ chercheuse indépendante en médiation muséale
}

RÉSUMÉ. II existe plusieurs offres de visite des musées, et en particulier des musées de sciences et de techniques. Les plus nombreuses sont des discours "savants " car s'adressant à l'intellect. Ils supposent généralement un bagage culturel et scientifique minimal qui intimide et détourne certains publics. D'autres offres peuvent prendre la forme de visites dansées, plus incarnées. Cependant, soit elles maintiennent le visiteur dans un rôle de spectateur, soit elles lui font interpréter une danse qui présente les sensations éprouvées par le chorégraphe face aux œuvres.

Cet article présente une autre approche, dite "sensible ", qui propose de mettre à disposition du visiteur les outils du chorégraphe pour lui permettre de créer sa propre interprétation des œuvres. Il décrit la méthode de conception de ces visites, les notions sur lesquelles elle se fonde, et son adaptation à la réalisation d'un "récit sensible ", forme de visite mêlant discours savant et approche sensible.

Les avantages et les limites de ce récit sensible sont présentées en conclusion. La mise en œuvre de l'approche sensible au sein des musées des beaux-arts semble montrer qu'il dispose d'un fort potentiel. Des expérimentations dans des musées scientifiques et techniques permettraient de le confirmer.

ABSTRACT. Several kinds of visit exist for museums and especially for science and technology museums. Most of them are "clever" discourses, built for the intellect. They generally require a minimal cultural and scientific background that intimidates and dissuades some audiences. Some others take the form of dance visits, more embodied. But all of them keep the visitor in a role of spectator or in a role of a performer who dance what the choreographer feels when facing at the works.

This article exposes a new approach, called "sensitive", which give to the visitor the choreographers' tools to let him create his own interpretation of the artworks. It explains the way to build these visits, the notions which fond it, and its adaptation to conceive a "sensible narrative", visit mixing clever discourse and sensitive approach.

Sensible narrative's advantages and limits are presented in conclusion. The implementation of the sensible approach in Fine Arts museums seems to show that this sensible narrative has a great potential. Experiments in science and technology museums would confirm it.

MOTS-CLÉS. science en mouvement, chorégraphie, médiation, musée, lien sensible.

KEYWORDS. science in motion, choreography, cultural mediation, museum, sensitive link.

Les musées conservent des œuvres d'art, des instruments scientifiques, des objets techniques qui possèdent une haute valeur culturelle, historique et scientifique. Cependant, cette muséalisation les retire de leur environnement, les « déshumanise ». Afin de les resituer dans la société qui les a vu apparaître, une mise en contexte et un récit sont conçus qui explique également le plus souvent l'intérêt scientifique ou technique de ces œuvres. Mais cette approche, que nous qualifierons de savante, propose des discours qui sont écrits autour de connaissances qui ne sont pas toutes fournies par l'exposition, discours qui sont alors reconstruits par les visiteurs eux-mêmes uniquement en fonction de leurs savoirs et de leur histoire ${ }^{1}$. De plus, cette approche savante s'adresse à l'intellect. Or, les recherches sur les profils des visiteurs ${ }^{2}$, notamment dans les musées de sciences et

\footnotetext{
${ }^{1}$ Schmitt, Daniel, Expérience de visite et construction des connaissances : le cas des musées de sciences et des centres de culture scientifique. Thèse, Sciences de l'information et de la communication, Université de Strasbourg, 2012, p 333.

${ }^{2}$ Falk John.H., Dierking, Lynn.D, The Museum Experience Revisited. Routledge, 2016.

(C) 2021 ISTE OpenScience - Published by ISTE Ltd. London, UK - openscience.fr 
techniques ${ }^{3}$, montrent que les intentions de visites sont variées et que tous ne viennent pas pour apprendre. C'est pourquoi de nouveaux principes de muséologie voient le jour, proposant des passerelles entre la culture exposée et celle du visiteur qui ne possède pas toujours toutes les clés pour comprendre ${ }^{4}$, s'adressant à tous ses sens, afin de lui permettre d'accéder aux œuvres comme il accède au monde.

La danse peut être une de ces passerelles, car elle apporte une approche sensible aux œuvres. De nombreuses expériences existent mais peu dans les musées de sciences et de techniques. Est-ce dû à la nature de ces musées ? En effet, comme l'a constaté Daniel Schmitt, les attendus des visiteurs dans chaque type d'institution patrimoniale sont différents : dans les centres de science, on apprend, dans les zoos, on compare et dans les musées d'art, on est impressionné ${ }^{5}$. Cette attente d'une approche savante dans les musées des sciences peut expliquer leur difficulté à attirer des publics qui, s'ils ne se considèrent pas aptes à comprendre, ne viendront pas. D'où l'idée de mêler l'approche sensible et l'approche savante, et de créer une relation personnelle aux œuvres, de la rendre vivante, de la mettre en mouvement.

Cet article propose une méthode de conception de ce double récit. Ce travail, en cours, s'appuie sur les recherches que Micheline Lelièvre mène depuis vingt ans, d'abord dans le paysage, puis au travers de conférences dansées, notamment des conférences scientifiques avec Damien Schoëvaërt, biologiste, et enfin dans divers musées d'art (Musée du Luxembourg Paris, Musée Malraux au Havre, pour le Centre des Monuments Nationaux $)^{6}$. Après avoir positionné cette recherche par rapport aux autres expériences dansées menées au sein des musées, nous exposons sa méthode de conception de l'approche sensible fondée sur sa définition des notions de chorégraphie et d'interprétation. Puis, nous détaillons notre proposition d'hybridation de son approche avec le discours savant. La conclusion décrit les atouts que ce récit savant et sensible peut apporter aux musées des sciences, ses limites et les travaux à mener pour les valider scientifiquement.

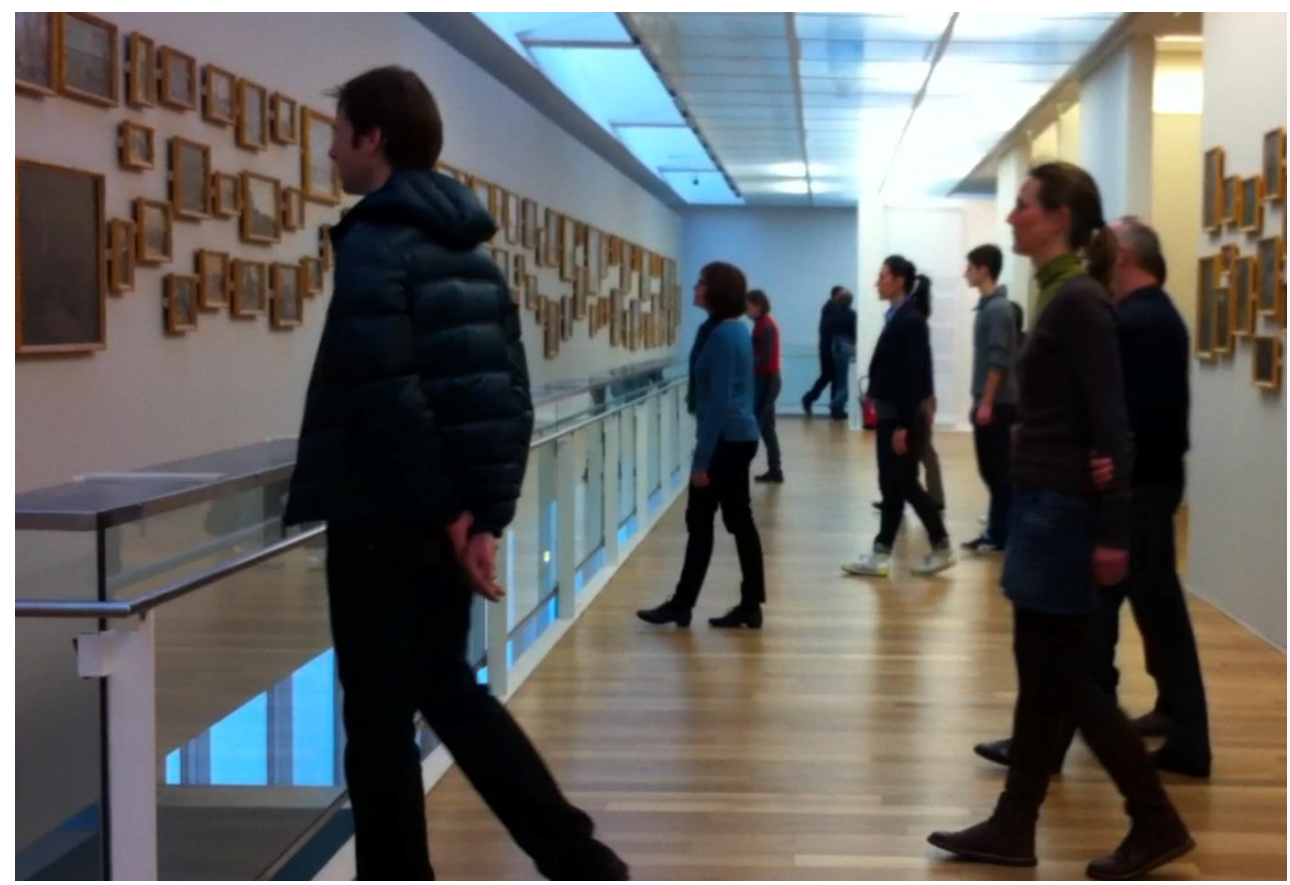

Figure 1. Approche sensible au musée Malraux du Havre (voir de loin, voir de près)

\footnotetext{
${ }^{3}$ Schmitt, Daniel, Op. Cit.

Rieuf, Vincent. Enrichir l'expérience muséographique du visiteur : Expériences, passages et engagements. In : Au-delà de l'art et du patrimoine. Janvier 2017.

${ }^{4}$ Gélinas, Dominique, Le sensorium synthétique : réflexion sur l'utilisation de l'expographie immersive numérique et muséale, Conserveries mémorielles. Revue transdisciplinaire de jeunes chercheurs, no \#16, sept. 2014. §46

${ }^{5}$ Schmitt, Daniel, Op. Cit. p 332

${ }^{6}$ Lelièvre, Micheline. Une chorégraphie au musée ? 2015. http://micheline.net/?page_id=48

(c) 2021 ISTE OpenScience - Published by ISTE Ltd. London, UK - openscience.fr 


\section{La danse au sein des musées}

La danse pénètre dans les musées de multiples façons. Elle peut être objet d'exposition, élément de médiation ou renouvellement de la visite accompagnée. Elle peut avoir pour objectif sa propre promotion ou, au contraire, se mettre au service d'un autre discours.

D'après Eloïse Guénard, l'idée d'exposer la danse aurait ressurgi « violemment » dans le courant des années 80 lorsque le milieu des danseurs s'est trouvé confronté au sida et à ses ravages. La volonté de conserver ce qui est par essence éphémère aurait conduit certains à patrimonialiser la danse par des chorégraphies dédiées à son histoire ou par des expositions rétrospectives ${ }^{7}$. D'autres initiatives ont valorisé les liens qui unissent danse et arts graphiques, comme l'exposition « Danser sa vie $»^{8}$. Dans l'un ou l'autre de ces cas, la danse apparaît soit comme le sujet de l'exposition, présenté grâce à des médias traditionnels : images, vidéos, textes, soit comme outil de médiation réflexif, c'est à dire de présentation de sa propre discipline.

Mais, la danse s'est également mise au service du musée ou de ses œuvres. Les premières expérimentations dans ce domaine avaient pour objectif de faire sortir l'interprétation dansée du théâtre où elle était habituellement présentée ${ }^{9}$. Intermède poétique visant à rendre vivant le musée quelque fois vécu comme un mausolée ${ }^{10}$, ou composition spécifique pour expérimentation ${ }^{11}$, la danse y était plutôt vécue comme un spectacle. Il apparut alors nécessaire de réfléchir à la place du visiteur, non nécessairement spectateur, et de créer un dialogue fécond entre la danse et les éléments d'art visuel présentés au sein de l'exposition ${ }^{12}$.

Les approches retenues sont de deux formes, selon le degré d'interactivité du visiteur. La première est la médiation, qui peut être uniquement sensible ou plus explicative. Dans le premier cas, la danse propose une interprétation des sensations vécues par le chorégraphe, sans explication. Ainsi, les danseurs du projet européen «Dancing Museums » s'exposent librement au public : ce dernier déambule dans les galeries et peut s'arrêter s'il le désire, et durant le temps qu'il désire, pour assister à la représentation ${ }^{13}$. D'autres propositions s'intègrent dans le cadre d'une visite particulière où le visiteur assiste à l'ensemble de la représentation. Ainsi la médiation explicative alterne prise de parole et danse. La parole explicite le ressenti de l'interprète vis-à-vis du tableau et par la même l'interprétation qu'il en effectue ensuite. Celle-ci peut être plus ou moins didactique, comme dans la proposition d'Aurélie Grandit ${ }^{14}$. Le visiteur y est toutefois spectateur, il n'est aucunement actif, au contraire de la danse participative. A l'occasion d'un atelier ${ }^{15}$ ou d'une visite accompagnée ${ }^{16}$, le visiteur interprète une danse, ou participe à l'élaboration d'une danse coopérative. Dans les deux cas, il est guidé par les instructions d'un danseur-chorégraphe ${ }^{17}$, autrement dit par les sensations éprouvées et les liens tissés par ce dernier avec les œuvres.

\footnotetext{
${ }^{7}$ Guénard, Eloïse, DE LA DANSE AU MUSEE AU MUSEE DE LA DANSE. Ecosystème, 2 (1), 2019, 35-42. p 35 https://do.org/10.7502/1062561ar

${ }^{8}$ https://www.centrepompidou.fr/fr/programme/agenda/evenement/cBG6ez

${ }^{9}$ Fournié, Fanny,. Des chorégraphes dans les musées d'art : le cas de Xavier Le Roy, Culture \& Musée, 27|2016, 123-128, p 124

${ }^{10}$ Bishop, Claire, Danse in the Museum. Publications and Research. CUNY. 2014, p 72

${ }^{11}$ Guénard, Eloïse, Op. Cit., p 38

${ }^{12}$ Bishop, Claire, Op. Cit., p 72

${ }^{13}$ https://www.creativeeuropeuk.eu/funded-projects/dancing-museums-democracy-beings

${ }^{14}$ https://vimeo.com/19248064

${ }^{15}$ https://www.louvre.fr/ateliers/les-nocturnes-du-vendredi-danses

${ }^{16} \mathrm{https://m.musee-orsay.fr/fr/en-famille/article/la-danse-a-loeuvre-visite-dansee-50644.html?eTime=1604829600}$

${ }^{17}$ Hayes, Marisa, Recherche sur la danse au musée, entretien avec Claire Bishop, Repères, cahier de danse, 2017/1-2 ( $n^{\circ} 38-39$ ), p. 38a-41a, p 38. DOI : 10.3917/reper.038.0038a. URL : https://www.cairn.info/revue-reperes-cahier-de-danse-2017-1-page- 
L'objectif de l'introduction de la danse au musée est de faire vivre l'exposition autrement, de créer un nouveau type de médiation entre le visiteur et l'œuvre, non uniquement savant. Cette démarche répond aux demandes d'ouverture du monde de la danse à d'autres spectateurs et d'autres lieux mais également aux études de public réalisées dans les musées indiquant qu'expérience, curiosité ou nécessité de se ressourcer constituent les uniques attentes de certains visiteurs ${ }^{18}$. L'importance de l'expérience du visiteur, qui n'est pas un spectateur, a été perçue par le monde de la danse et fait partie des études du projet « Dancing Museums » et de la réflexion de Claire Bishop. Il s'agit alors de concilier les comportements différents du spectateur et du visiteur : comment résoudre les contradictions entre la temporalité de la danse et la liberté du visiteur à déambuler dans les galeries ? Comment lui donner un rôle actif dans l'interprétation habituellement contemplative qu'il a de l'œuvre ? Les propositions actuelles oscillent entre attribuer malgré tout au visiteur un rôle de spectateur ou lui donner un rôle d'interprète. Aucune ne lui permet de construire son propre lien personnel, de se forger sa propre interprétation sensible. Le lien qui se crée entre le visiteur et l'œuvre est toujours un lien par procuration dans la mesure où les sensations qu'il expérimente par la danse sont celles d'un ou d'une autre. Or, si pour l'approche savante, le discours est reconstruit par le visiteur en fonction de ses connaissances et de ce que lui offre le musée ${ }^{19}$, il nous semble important qu'il en soit de même pour l'approche sensible. Comment lui donner les moyens de créer son propre lien avec l'objet par la danse ? C'est à cette question que ce travail de recherche a souhaité apporter une réponse.

\section{La posture de chorégraphe, points de départ et méthode.}

Dans son livre, Suzanne Langer ${ }^{20}$ soutient que la signification de l'art devrait être trouvée dans l'objet d'art lui-même, tel qu'il se présente à notre conscience, et non dans ce qu'il serait supposé représenter ou signifier. Elle affirme que si les hommes ont du mal à saisir cela dans les sociétés occidentales, c'est parce qu'ils sont tellement habitués à considérer l'art comme une représentation de quelque chose que leurs réactions aux représentations elles-mêmes se confondent toujours avec leurs réactions devant ce qu'elles sont supposées signifier. La proposition effectuée ici se situe à l'endroit où le visiteur est au plus proche de sa réaction première. Elle se sert en partie des outils de l'interprète pour créer du lien entre des œuvres, des lieux et un public. Au lieu de montrer une seule interprétation, qu'elle soit dansée ou savante, l'idée est d'utiliser ces outils pour donner des pistes au visiteur pour qu'il ne soit pas spectateur mais acteur, uniquement guidé pour se rapprocher de ses propres perceptions, sensations, imaginaires. L'objectif est de le mettre en capacité d'interpréter ce qui est devant lui par lui-même, ou en partant de sa propre expérience. Au cœur de cette démarche se trouvent les notions de chorégraphe et d'interprète. C'est pourquoi, nous exposons en premier lieu les définitions que nous avons adoptées dans le cadre de cette recherche, avant de décrire la méthode de conception de l'approche sensible.

\subsection{Notions de chorégraphie et d'interprétation}

Pour le commun des mortels, et c'est légitime de le penser, un danseur danse, c'est-à-dire qu'il produit des mouvements qui, dans leur proposition, leur organisation et leur interprétation ne sont pas quotidiens. Être chorégraphe est une posture différente de celle d'être interprète.

Chorégraphier, c'est organiser des situations, créer de l'inattendu parce que le point de vue va être inhabituel, c'est penser au « voyage » que le chorégraphe va tisser à travers le lieu, les œuvres. Puis c'est convoquer les compétences de l'interprétation pour nourrir cette construction. Une visite se conçoit, avec une cohérence par rapport à un projet. Le projet peut être de mettre l'accent sur tel ou tel point de vue, telle émotion... la visite se construit à partir de tous ces éléments.

\footnotetext{
${ }^{18}$ Falk John.H., Dierking, Lynn.D, Op. Cit.

${ }^{19}$ Schmitt, Daniel, Op. Cit. p 317

${ }^{20}$ Langer Suzanne, Philosphy in a New Key: a Study in the Symbolism of Reason, Rite, and Art, Harvard University Press, 1957 
La place de l'interprétation est autre. On pourrait dire que ce qui fait danse est l'interprétation de mouvements. L'interprète, c'est celui ou celle qui se trouve « entre », entre un auteur, en ce sens que c'est l'auteur qui propose un matériau de départ, qu'il s'agisse d'une idée, de mouvements, et un public. L'interprète est celle ou celui qui va donner du sens, qui va traduire peut-être, en tous cas qui va avoir une posture de liaison au service d'un propos, d'une proposition d'un auteur. Interpréter signifie que l'on a développé des compétences particulières, des outils : s'imprégner d'un projet, d'un propos, recevoir des consignes et leur donner vie tout en respectant le point de vue de l'auteur, nourrir cela de qualités personnelles. L'interprète est toujours sur un fil, entre la proposition de l'auteur et sa richesse personnelle. La plupart du temps il ou elle réalise cette interprétation par le mouvement, dans le cadre d'une production qui s'appelle une chorégraphie, une performance.

\subsection{Conception d'une approche sensible}

Chaque personne peut être considérée comme interprétant constamment son environnement, ses relations, son rapport aux œuvres, aux lieux, aux autres. Ce que l'artiste va apporter, c'est de faire prendre conscience de cette interprétation personnelle, d'avoir des pistes pour percevoir d'où naissent ces sensations, tout cet invisible qui nous sert à vivre, à communiquer, échanger, et de mettre tout cela en relation avec le corps, le corps en mouvement, donc le corps vivant. Ce sont les compétences croisées, de chorégraphe et d'interprète qui permettent de faire vivre ces visites à des publics divers. La danse a donc sa place, que ce soit dans les musées ou les lieux auxquels elle donne une vie particulière, sensible, mais pas spectaculaire.

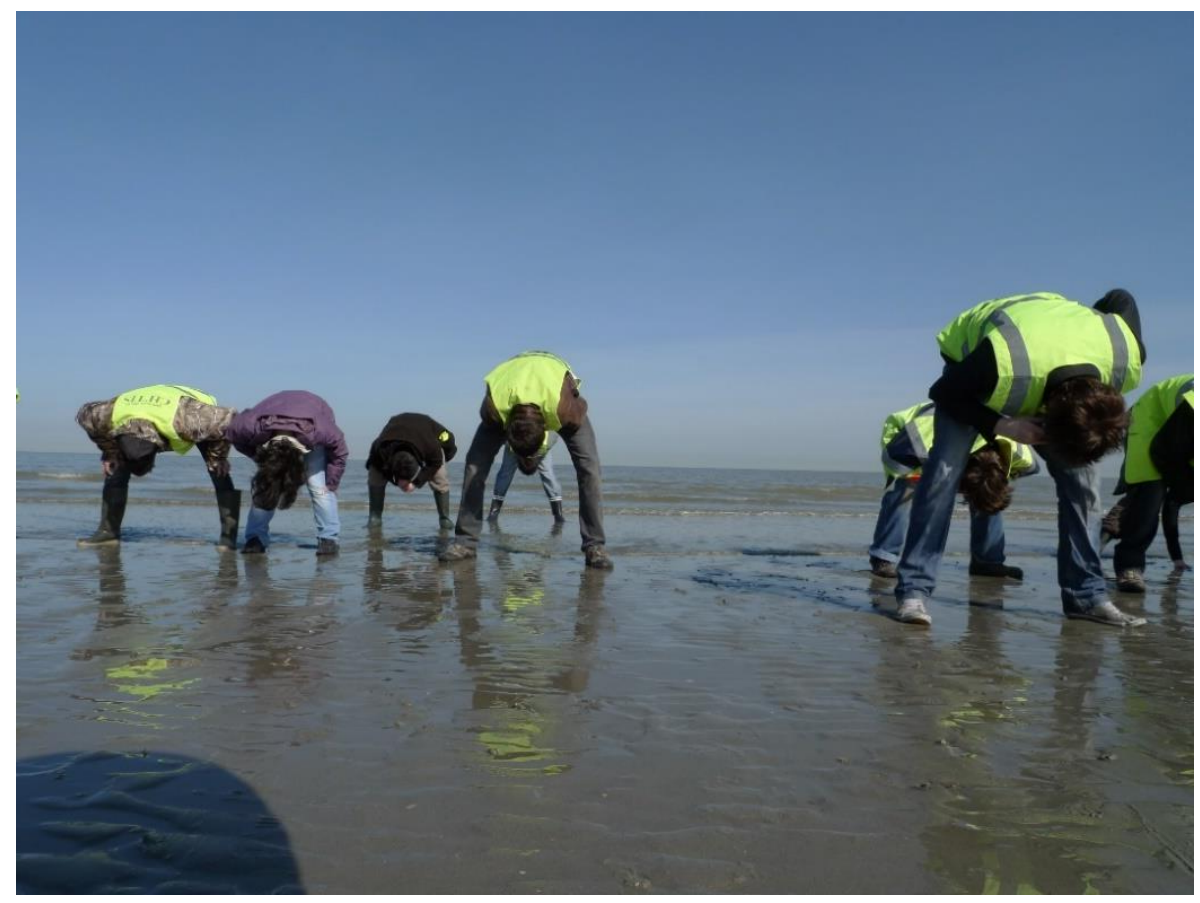

Figure 2. Percevoir le paysage, voir le monde à l'envers.

Il est important de préciser que ceci est la manière de procéder propre à l'auteure chorégraphe Micheline Lelièvre. Il y a d'autres façons de creuser cette question associée à d'autres outils utilisés. En fait, ils relèvent de chaque créateur. L'important est d'être en accord avec soi-même pour construire ces rencontres avec des œuvres, des objets, des scénographies, des paysages. De son point de vue, c'est là son rôle de chorégraphe, d'artiste : de partager avec des visiteurs, ces moments dans les musées, les paysages, les villes....

Dans le cadre de cette proposition, la présence de la chorégraphe est de l'ordre de la performance. Elle est dans un acte artistique, elle crée de l'imaginaire dont s'empare le spectateur, le visiteur, chacun comme bon lui semble, autour d'une œuvre, d'objets, de lieux..., même si la chorégraphe ne danse quasi jamais dans ce contexte. Elle énonce des consignes, qui sont autant d'outils qui servent 
à ouvrir les portes de l'imaginaire, du ressenti, de la présence à soi-même et au monde qui nous entoure, autant d'invitation à les exprimer.

La notion de présence est le cœur de la question. Elle consiste en une attention, à soi, aux autres, à l'espace, à l'environnement et comment ces différents éléments circulent. C'est une attention au flux, au mouvement. C'est une mise en disponibilité pourrait-on dire, une ouverture pour que l'imprévu, la surprise arrive. C'est pourquoi cette proposition n'est pas réalisée au sein d'un atelier au cours duquel les visiteurs seraient amenés à un résultat. Les consignes, ces outils, sont des prétextes à créer une disponibilité, une présence : être là, présents à ce qui advient, à ce que l'on ressent, à ce que l'on voit, à ce que l'on ne voit pas....

Pour arriver à faire expérimenter ces sensations, la méthode utilisée est à deux niveaux. Le premier est une longue phase de préparation, d'examen des données qui sont proposées : aller chercher quel est le fil directeur d'une œuvre, d'une scénographie, d'un ensemble d'objets (selon les circonstances) ; aller observer les qualités qui émergent, les organisations d'espaces, les renvois symboliques sensibles; identifier une fonction.... Par exemple, dans le laboratoire de Lavoisier, exposé au Musée des arts et métiers, l'artiste s'est intéressée à la position des meubles, des objets et instruments scientifiques, à leur dimension, leur poids. L'important est de garder l'esprit ouvert, de laisser affleurer les ressentis, les idées, pour pouvoir ensuite créer une proposition.

Ensuite, le second point consiste à construire un parcours avec tout ce matériau qui s'est déposé comme un limon dans l'esprit de la chorégraphe. Elle met alors en œuvre les compétences particulières de son métier car il s'agit de construire une proposition en surimpression, ou en parallèle, selon les circonstances, avec des objets, des œuvres, dans une cohérence d'espace, celui de la déambulation, puisque c'est généralement la forme que prennent les visites. L'idée est d'explorer, d'être attentif à l'installation muséale en partant de son propre corps et des diverses manières d'être investi dans celui-ci, d'y être présent, de faire fonctionner son imaginaire en étant guidé par quelqu'un d'expérimenté dans ce type d'exploration sensorielle. Concrètement, dans le cadre de l'expérimentation dans le laboratoire de Lavoisier, l'artiste a suggéré aux visiteurs d'observer le laboratoire, d'en répertorier les différents objets, meubles.... puis de s'imaginer en train de circuler au travers de manière assez libre (passer sous la table ou par-dessus...). Ensuite, elle leur proposa de changer de point de vue, en modifiant leur place dans l'espace pour voir le laboratoire autrement, puis de l'envisager à partir d'un autre niveau (à quatre pattes, sur la pointe des pieds...). Elle a alors ajouté des consignes de qualité de mouvement: se sentir lourd, léger, voler, circuler comme une souris....

Cette méthode ne servirait pas à autre chose qu'à concevoir un atelier si elle ne s'accompagnait d'une posture, d'un état, qui va déterminer une qualité de présence pour soi et pour les autres et donc les mettre, du moins espère-t-on, eux aussi dans cet état de présence, de disponibilité, d'ouverture à ce qui arrive et qui peut aussi surprendre, amuser, étonner... La chorégraphe crée une situation et chacun s'empare de ses propositions comme bon lui semble, comme il se sent. Il n'y a pas d'obligation à se mettre en mouvement, il n'y a pas de résultat à obtenir, hormis celui d'avoir pris le temps d'être là, sans projection, sans jugement, au présent donc. Il est donc capital que la chorégraphe soit dans une posture et un état de disponibilité, qui est aussi une fragilité. Il faut dans le même temps savoir ce que l'on fait et n'avoir aucune idée préconçue sur ce que cela va avoir comme effet sur les autres, donc accueillir la situation présente. C'est aussi ce qui est ressenti sur scène, c'est pour cela que ces interventions peuvent être appelées «performances ». Il s'agit d'une présence artistique, qui ne saurait se substituer à une médiation, à un savoir savant, mais qui peut venir en complément ou plutôt en supplément de sens pour enrichir l'expérience du visiteur au musée. 


\section{Création d'une relation sensible et savante}

Une expérience de cette supplémentarité a été imaginée dans le cadre d'une médiation scientifique et technique. L'idée était d'associer, d'interpénétrer les deux approches, savante et sensible, en construisant un récit. Partant du principe que toute pensée peut être transformée en image mais également en mouvement interne et perception du sensible ${ }^{21}$, l'idée n'est pas d'opposer approche savante et approche sensible mais de les mettre en synergie afin d'offrir au public la perception des problématiques ou des informations selon deux angles différents pensés en dialogue. Il ne s'agit pas de faire observer, apprendre ou comprendre quelque chose mais de permettre au visiteur de créer aussi un lien sensoriel, émotionnel avec l'œuvre, invitant tous les sens : auditif, visuel, kinesthésique.

Notre processus de conception du protocole est constitué de cinq étapes, reprenant les principes de construction de récit de Lindley ${ }^{22}$. Tout débute par la mise en commun de l'ensemble des propositions que nous pouvons faire à l'autre : la médiatrice scientifique expose les connaissances associées aux objets, à leurs auteurs, à leur contexte historique et aux espaces du musée. Elle explique également la façon de les décrire. La chorégraphe parle de son art, sa façon de créer un "état de présence" au monde, les éléments sur lesquels elle peut établir ses propositions, ce qui l'intéresse plus particulièrement dans la description des objets, les performances qu'elle a déjà proposées. Cette première étape permet de construire une culture commune aux deux parties, facilitant les étapes suivantes.

La seconde étape consiste à trouver la thématique de la proposition, de l'histoire à raconter, à partir des objets du musée. Cette phase est celle d'une étude savante pour la médiatrice. Le travail de la chorégraphe est de prendre connaissance de l'ensemble des objets, d'en comprendre les relations. Puis chacune expose à l'autre ses recherches. Cette étape correspond au premier niveau de la méthode de conception de l'approche sensible. Dans le cadre de nos travaux, elle a porté, entre autres, sur les premiers automates et les premières machines à calculer. Alors que la médiatrice s'intéressait et expliquait la filiation des machines et leur principe de fonctionnement, la chorégraphe était sensible à la complexité du métier à tisser, aux sensations associées aux mouvements extrêmement variés des mécaniques, à la multitude de composants techniques assemblés, aux engrenages de la Machine à calculer de Pascal, etc., ses trouvailles. A l'issue de nos échanges, le thème de l'algorithme nous a paru riche en possibilités pour les deux approches.

La troisième étape est la définition des éléments qui constituent les points saillants du récit, pour leur rôle dans la médiation savante et leur apport pour le parcours sensible, notamment dans les possibilités de sollicitations de l'imaginaire du visiteur qui lui permettront de créer sa propre relation avec la machine. Ainsi, pour notre travail sur la notion d'algorithme, la calculatrice de Pascal (1645) et la machine à calculer de Bollée (1889) sont apparus comme deux objets structurants d'un point de vue technique et d'un point de vue sensible. La première, une des toutes premières machines à calculer, présente un principe d'utilisation simple autour d'une unique fonction visible, l'addition, conçue à l'aide d'engrenages, à fort potentiel sensible. La seconde est un exemple précoce de machine à multiplier. Pour obtenir le résultat de la multiplication, une suite conséquente de manipulations est nécessaire mettant en jeu un nombre important de manettes, engrenages et manivelles.

Vient ensuite la structuration du récit. Elle s'effectue conjointement avec la médiatrice et la chorégraphe. Le rôle de la première est de détailler le lien entre les objets et le sujet de la médiation. Elle fournit les informations indispensables à leur compréhension. La chorégraphe propose des manières d'entrer en relation avec leur fonction, de rendre sensible ce qui ne le semble pas au

\footnotetext{
${ }^{21}$ Lelièvre, Micheline, Du calcul aux algorithmes, une visite dynamique, proposition de médiation, Note d'introduction, 2018.

${ }^{22}$ Lindley, Craig A. Story and Narrative Structures in Computer Games. In Bushoff, Brunhild. (ed). Developing Interactive Narrative Content: sagas/sagasnet reader. 2005, Munich: High Text. 
premier abord. Cette quatrième étape constitue le second niveau de la méthode de conception du parcours sensible. Tout comme la médiation savante, la proposition sensible progresse au fur et à mesure de la visite pour tisser des liens de plus en plus nombreux entre le visiteur, sa perception, son imagination et sa compréhension. Ces liens se créent grâce à des suggestions de la chorégraphe comme «se mettre à la place de l'inventeur », « circuler en imagination dans une machine », «créer du rythme dans sa marche, même de façon imperceptible », «changer d'angle de vision ». D'autres indications sont en relation avec l'explication savante. Par exemple, la simplicité de la machine à calculer de Pascal, opérant une multiplication par la répétition de l'addition, sera présentée par la répétition d'un même mouvement. Elle se distinguera de la pluralité de postures proposées pour rendre sensible la mise en œuvre de la multiplication avec la machine de Bollée. Les suggestions de la chorégraphe permettent de créer une relation concrète avec des informations abstraites, relations qui ne sont cependant jamais explicitées au visiteur.

L'ultime étape est la matérialisation du récit sous sa forme définitive : un dialogue entre les deux discours, savant et sensible. Le style et le rythme de celui-ci sont travaillés. Afin de donner une dynamique au discours, des textes courts, des mots simples, sont privilégiés. Des énumérations rapides de propositions d'activités sollicitant l'imaginaire ("montez, descendez, sautez, culbutez") permettent de créer du rythme dans la narration. Les descriptions des dispositifs techniques sont limitées aux éléments nécessaires pour comprendre la thématique abordée. Les phrases écrites sont volontairement inclusives, employant le "nous" plutôt que le "vous" pour inciter le visiteur à se joindre aux autres, à ne pas se sentir seul mais au contraire à intégrer une communauté, créant une relation de confiance entre le narrateur et le visiteur.

\section{Conclusions}

Ce récit, que nous appellerons "récit sensible" est construit autours d'événements à la fois réels (les objets) et imaginaires (les propositions de rencontres avec ces objets qui sont autant de portes vers l'imaginaire du visiteur). Il mêle des approches intellectuelles mais également sonores, visuelles, spatiales, par la prise en compte par le visiteur de l'espace autour de lui, et kinesthésiques, par les gestes et les mouvements qui lui sont suggérés de réaliser. Quels sont les apports réels de ce récit sensible? Quelle expérience en tire le visiteur?

L'idée de ce récit sensible est d'explorer, d'être attentif à l'installation muséale non pas à partir de celle-ci mais à partir de ses propres sensations, de son propre corps, des diverses manières d'être investi dans celui-ci, d'y être présent, de faire fonctionner son imaginaire en étant guidé par quelqu'un d'expérimenté dans ce type d'exploration sensorielle. Les indications suggérées par l'artiste proposent au visiteur de vivre ou d'imaginer des sensations en relation avec les œuvres, son environnement ou les propositions de médiation. Elles créent un "état de présence" à soi et aux autres, aux objets, à l'environnement, tissant des liens entre l'intellect du visiteur, ses sens et l'approche savante. En travaillant sur les perceptions, en incitant à ressentir des sensations imaginaires, des dynamiques invisibles, cette démarche permet de rendre sensibles, et donc concrètes, des informations concernant les objets techniques présentés, de s'intéresser aux objets autrement, de les relier, si le visiteur le souhaite, à un savoir. Elle rend alors concret ce savoir. Le récit sensible remet ainsi l'œuvre dans une réalité humaine et la resitue dans un vécu.

Il n'opère cependant que si le visiteur adhère à la proposition. La chorégraphe ne fait que suggérer, mais le visiteur est libre de suivre ses indications ou pas. C'est une des limites de cette approche. Une seconde est le possible abandon, par le visiteur, de sa liberté de déambulation. Il peut se laisser guider par le rythme proposé par la chorégraphe. Cependant, et c'est l'un des points fondamentaux de cette proposition, cette dernière se met dans un état de présence qui lui permet de demeurer attentive au comportement des visiteurs et d'adapter ses indications à leur état et à leur rythme. 
Prendre le temps d'observer tranquillement et de façon ludique ce qui se tisse entre soi et l'extérieur modifie l'approche que l'on peut avoir des objets, des installations dans un musée mais également des visites de musées elles-mêmes. Cette approche ne demande pas de connaissances particulières. Les propositions initiées par la chorégraphe ont pour objectif de mettre le public qui le souhaite en relation avec des œuvres ou des objets à partir de leurs sensations et perceptions personnelles. C'est une entrée en matière poétique et sensible qui n'est en rien concurrentielle d'un propos scientifique. Au contraire, cela ouvre le champ d'une collaboration fructueuse entre la science et l'art. Une fois la sensibilité et la curiosité éveillées, le propos savant est là pour ancrer les connaissances. Entrer en résonance avec une œuvre ou un objet ouvre la porte au désir. L'incarnation est complémentaire d'une approche purement intellectuelle, elle fournit un point d'entrée attirant pour les visiteurs intimidés par les connaissances qu'ils supposent nécessaires pour imaginer se distraire dans un musée technique.

Afin de finaliser ce travail, des expériences sont à mener au sein de musée, et en particulier de musée des techniques, pour évaluer l'apport de ce type de récit savant et sensible. Si le récit sensible n'a pas été testé en tant que tel dans un musée technique, le parcours sensible, sans la partie médiation donc, fut mis en œuvre dans le cadre de musées de beaux-arts ${ }^{23}$. L'expérience a prouvé qu'un tiers des participants à la visite sont des personnes qui ne viennent pas d'habitude au musée et que cette mise en relation permettait à un certain nombre de personnes de se rapprocher des œuvres ou des objets par-delà leurs peurs ou leurs présupposés d'incompétence. Ce rapport sans complexes, sans a priori, et donc sans frein, facilite la création d'un intérêt pour les œuvres et conduit à l'envie d'en savoir plus : environ la moitié des participants retourne dans le musée pour visiter par ellemême, directement après la performance ou quelque fois plus tard. Une évaluation pourrait ainsi porter sur le nombre de personnes venues spécifiquement dans les musées de sciences et de techniques pour vivre cette expérience, sur l'image véhiculée par ces visites pour ce type de musée, sur la satisfaction des visiteurs et leurs relations aux œuvres techniques : comment les perçoivent-ils avant et après la visite ? est-ce que leur approche a évolué ? Ces changements d'attitude témoigneraient d'un rapport différent avec l'œuvre, peut-être plus complet. Ils indiqueraient, voire prouveraient, que le récit savant et sensible constitue une autre porte d'accès aux œuvres et que la danse a toute sa place pour une meilleure rencontre, plus complète et moins intimidante, avec les œuvres des musées de sciences et techniques. 